01

\title{
Эффекты амплитудно-зависимого внутреннего трения В низкочастотном стержневом резонаторе из отожженной поликристаллической меди
}

\author{
(C) В.Е. Назаров, ${ }^{1}$ А.Б. Колпаков ${ }^{2}$ \\ ${ }^{1}$ Институт прикладной физики РАН, \\ 603950 Нижний Новгород, Россия \\ ${ }^{2}$ Нижегородский государственный университет им. Н.И. Лобачевского, \\ 603022 Нижний Новгород, Россия \\ e-mail: v.e.nazarov@appl.sci-nnov.ru
}

Поступило в Редакцию 1 февраля 2021 r.

В окончательной редакции 16 апреля 2021 г.

Принято к публикации 17 апреля 2021 г.

\begin{abstract}
Проведены экспериментальные и теоретические исследования эффектов амплитудно-зависимого внутреннего трения в низкочастотном стержневом резонаторе из отожженной поликристаллической меди. Представлены результаты измерений нелинейных потерь и сдвига резонансных частот на первых трех продольных модах резонатора в килогерцовом диапазоне частот от 2 до $11 \mathrm{kHz}$. Аналитическое описание наблюдаемых эффектов проведено в рамках реологической модели и уравнения состояния микронеоднородной среды с насыщением гистерезисных потерь и релаксацией ее вязкоупругих дефектов. Из сравнения экспериментальных и аналитических результатов определены значения эффективных параметров гистерезисной нелинейности образца отожженной меди и их зависимость от частоты.
\end{abstract}

Ключевые слова: амплитудно-зависимое внутреннее трение, насыщение гистерезисных потерь, резонатор.

DOI: 10.21883/JTF.2021.09.51208.21-21

\section{Введение}

Исследования нелинейных волновых процессов в микронеоднородных средах, в частности в кристаллических твердых телах (металлах и горных породах), актуальны и интересны по многим причинам, главной из которых является их аномально высокая акустическая нелинейность $[1,2]$ по сравнению с однородными слабонелинейными материалами $[3,4]$. Со „структурной“ точки зрения, отличие микронеоднородных сред от однородных связано с тем, что микронеоднородные среды содержат различного рода нелинейные вязко-упругие дефекты (дислокации, зерна, трещины и т.д.), размеры которых малы по сравнению с длиной волны [5]. Сильная и, как правило, неаналитическая (гистерезисная, разномодульная, диссипативная и т. д.) нелинейность микронеоднородных сред определяется высокой по сравнению с окружающей однородной средой „сжимаемостью“ таких дефектов (для дислокаций - высокой подвижностью) и их соответствующей неаналитической нелинейностью. Релаксация дефектов, связанная с восстановлением их термодинамического равновесия, нарушаемого под действием упругой волны, приводит к тому, что нелинейность микронеоднородных сред становится частотнозависимой [2]. Однородные среды никаких дефектов не содержат, поэтому они обладают слабой безынерционной (частотно-независимой) аналитической (квадратичной и/или кубичной) упругой нелинейностью, обуслов- ленной слабым ангармонизмом межатомного (межмолекулярного) взаимодействия [3,4].

Закономерности нелинейных эффектов, возникающих при распространении и взаимодействии интенсивных упругих волн в средах с различными видами нелинейности (аналитической и неаналитической), качественно отличаются, что можно использовать для определения динамических уравнений состояния микронеоднородных сред, адекватно описывающих нелинейные волновые процессы в таких средах.

Уравнения состояния кристаллических твердых тел даже при относительно малых амплитудах деформаций, характерных для акустических волн, как правило, являются гистерезисными, что приводит к эффектам амплитудно-зависимого внутреннего трения (АЗВТ): нелинейным потерям и нелинейному дефекту модуля упругости [6-15]. Гистерезисная нелинейность кристаллов связывается с проявлением микропластичности [7], с движением дислокаций и их взаимодействием с примесными атомами [8-15]. Один из основных вопросов при изучении гистерезисных эффектов в различных кристаллах - это выявление их амплитудных и частотных зависимостей. Надежно установлено, что амплитудные зависимости эффектов АЗВТ в разных кристаллах, как правило, различны [14-18] и часто не соответствуют теоретическим [9-15]. Это определяется различной нелинейной динамикой дислокаций, зависящей от большого числа структурных характеристик поликристалла (типа решетки, функции распределения, плотности и 
вида дислокаций, концентрации вакансий, межузельных и примесных атомов, размеров зерна и т.д.). Более интересная и непонятная ситуация была связана с частотной зависимостью эффектов АЗВТ. В первых теоретических работах по АЗВТ полагалось, что гистерезисная нелинейность кристалла является безынерционной, и, следовательно, эффекты АЗВТ от частоты не зависят [7-15]. В начале, в не очень многочисленных экспериментальных работах по изучению эффектов АЗВТ в металлах $(\mathrm{Cu}, \mathrm{Zn}, \mathrm{Pb})$, наблюдались различные частотные зависимости, а именно падающие с ростом частоты [8], растущие с ростом частоты [19] и не зависящие от частоты $[17,20]$. На основе анализа результатов таких работ было решено, что имеющихся экспериментальных данных недостаточно, чтобы определенно ответить на вопрос о частотной зависимости эффектов АЗВТ.

В последовавших теоретических работах [21-23] был предложен механизм термо-активированного взаимодействия дислокаций с точечными дефектами, приводящий к частотной зависимости АЗВТ. Эти работы предсказывают уменьшение эффектов АЗВТ с ростом частоты. В других работах [24-26] идея термоактивированного отрыва дислокаций получила дальнейшее развитие в плане учета и влияния различных распределений точечных дефектов вдоль и около линии дислокации. В этих работах было показано, что гистерезисные потери, обусловленные термомеханическим отрывом, в низкочастотном диапазоне линейно растут с частотой, а в высокочастотном - уменьшаются.

В цикле экспериментальных работ [27-32] было показано, что в щелочно-галоидных кристаллах $\mathrm{LiF}$, $\mathrm{NaCl}$ (в диапазоне частот $5 \cdot 10^{-3}-5 \mathrm{~Hz}$ ), а также в некоторых металлах и сплавах $\mathrm{Zn}, \mathrm{Al}$ (в диапазоне частот $5 \cdot 10^{-3}-1 \mathrm{~Hz}$ ) и $\mathrm{Cu}, \mathrm{Ni}$ (в диапазоне частот $5 \cdot 10^{-3}-1 \mathrm{~Hz}$ и на отдельных частотах около $1 \mathrm{kHz}$ и $100 \mathrm{kHz}$ ) наблюдалось в основном уменьшение эффектов АЗВТ с ростом частоты. Для объяснения этих результатов в работах [28-30] предлагались реологические модели неупругой микропластической деформации и дислокационной амплитудно-зависимой неупругости, учитывающие термоактивированный характер взаимодействия и отрыва дислокаций от точечных дефектов. В этих работах было показано, что с ростом частоты амплитудно-зависимый дефект модуля упругости уменьшается, а декремент затухания вначале растет и достигает максимума, а затем уменьшается.

В некоторых металлах и сплавах $(\mathrm{Cu}[11,12], \mathrm{Pb}[20]$, $\mathrm{Cu}-\mathrm{Ni}$ [32], Al [33], In [34]) при увеличении амплитуды упругой волны имеет место насыщение гистерезисных потерь. В работах [6,35] были предложены гистерезисные уравнения состояния для микронеоднородных твердых тел с насыщением эффектов АЗВТ и проведено теоретическое исследование нелинейных волновых процессов в таких средах. В этих работах рассматривались соответственно безынерционное и релаксационное уравнения состояния с частотно-независимой и частотнозависимой гистерезисной нелинейностью, при этом в [6] было показано, что амплитудно-частотные зависимости эффектов АЗВТ, определяются гистерезисными вязкоупругими дефектами микронеоднородного твердого тела и их распределением по линейным (упругим и неупругим) параметрам.

Следует отметить, что установление амплитудночастотных зависимостей эффектов АЗВТ в различных кристаллах является актуальной задачей физики твердого тела и составляет основу для изучения динамики дислокаций под действием знакопеременных упругих напряжений и определения механизмов гистерезисной нелинейности кристаллических твердых тел.

В настоящей работе приводятся результаты экспериментальных и теоретических исследований эффектов АЗВТ (нелинейных потерь и сдвига резонансных частот) в низкочастотном стержневом резонаторе из отожженной поликристаллической меди. Измерения проводились на первых трех продольных модах резонатора в килогерцовом диапазоне частот от 2 до $11 \mathrm{kHz}$. Аналитическое описание эффектов АЗВТ проведено в рамках уравнения состояния микронеоднородной среды с насыщением гистерезисных потерь и релаксацией ее вязко-упругих дефектов [2,6]. Из сравнения экспериментальных и аналитических результатов определены значения эффективных параметров гистерезисной нелинейности образца отожженной меди и их зависимость от частоты.

\section{1. Схема эксперимента}

Эксперименты по изучению эффектов АЗВТ проводились со стержневым резонатором, изготовленным из отожженной поликристаллической меди $(99.97 \% \mathrm{Cu})$. Длина стержня $L=4.3 \cdot 10^{-1} \mathrm{~m}$, диаметр его поперечного сечения $-8 \cdot 10^{-3} \mathrm{~m}$. Стержень отжигался при температуре $600^{\circ} \mathrm{C}$, время отжига - около $2 \mathrm{~h}$. (Отжиг поликристаллической меди приводит к изменению ее микроструктуры, а именно к росту зерна и к уменьшению плотности дислокаций [36].) На рис. 1 приведены фотографии микроструктуры неотожженной и отожженной меди, полученные с боковых поверхностей неотожженного и отожженного стержней. Видно, что текстура неотожженной меди имеет „волокнистую“ структуру, ориентированную вдоль направления волочения (вдоль стержня), а при отжиге образуются зерна с характерными размерами от 20 до $100 \mu \mathrm{m}$.

Схема эксперимента изображена на рис. 2. К нижнему торцу стержня 1 приклеивался пьезокерамический излучатель накачки 2 для возбуждения в нем продольной низкочастотной (НЧ) стоячей волны; другая сторона излучателя 2 была приклеена к массивной металлической нагрузке 3 . К верхнему, свободному торцу стержня 1 приклеивался пьезокерамический акселерометр 5 для приема колебаний, создаваемых излучателем 2. Стержень 1 вместе с излучателем накачки 2 и нагрузкой 3 является акустическим резонатором с жесткой нижней $(x=0)$ и мягкой верхней $(x=L)$ границами; 

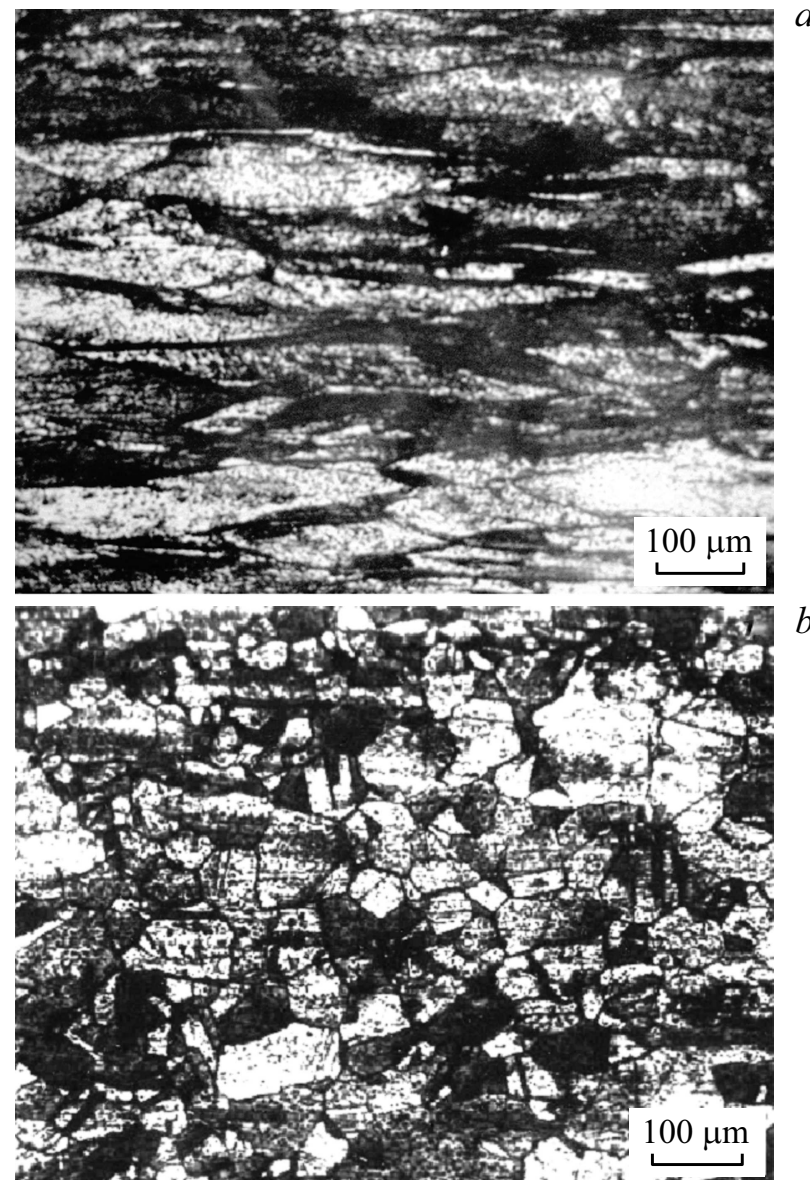

Рис. 1. Фотографии микроструктуры неотожженной $(a)$ и отожженной $(b)$ меди.

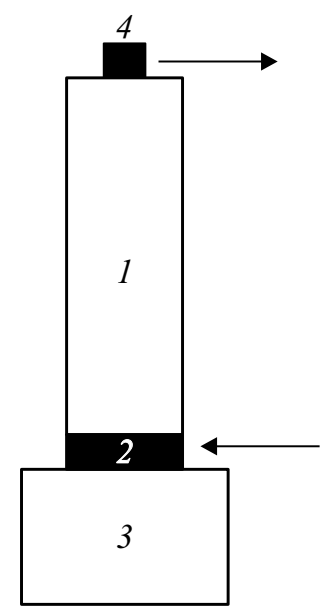

Рис. 2. Схема экспериментальной установки: $1-$ стержневой резонатор, 2 - излучатель накачки, 3 - массивная металлическая нагрузка, 4 - пьезокерамический акселерометр.

резонансные частоты $F_{p}$ его первых продольных мод пропорциональны нечетным числам $(1,3,5, \ldots)$ и при идеальных границах резонатора и без учета геометрической дисперсии фазовой скорости в стержне опреде- ляются выражением: $F_{p}=(2 p-1) C_{0} / 4 L, C_{0}-$ фазовая скорость НЧ продольной волны в стержне, $p$ - номер моды, $p=1,2,3, \ldots$ Для такого резонатора локальная амплитуда $\epsilon_{m}=\epsilon_{m}(x)$ деформации стоячей НЧ волны зависит от координаты $x$ и определяется выражением: $\epsilon_{m}(x) \approx \varepsilon_{m}\left|\cos K_{p} x\right|$, где $\varepsilon_{m} \approx K_{p} U_{1}-$ амплитуда деформации в резонаторе, $U_{1}$ - амплитуда смещения $U(x, t)$ на частоте накачки $F, K_{p} L=(2 p-1) \pi / 2$.

Линейные резонансные частоты $F_{p}$ и добротности $Q_{p}$ резонатора (при малой амплитуде возбуждения, когда нелинейные эффекты не проявляются) определялись по максимуму амплитуды колебаний резонатора и ширине $\Delta F_{0.71}$ его резонансной кривой на уровне от максимума: $Q_{p} \cong F_{p} / \Delta F_{0.71}$. Значения $F_{p}$ и $Q_{p}$ для первых трех продольных мод резонатора составляли: $F_{1}=2204 \mathrm{~Hz}$, $F_{2}=6447 \mathrm{~Hz}, \quad F_{3}=10697 \mathrm{~Hz}$ и $Q_{1}=450, Q_{2}=883$, $Q_{3}=578$. Небольшое отличие значений резонансных частот $F_{p}$ от соотношения $F_{p} \propto(2 p-1)$ связано с проявлением слабой дисперсии, обусловленной отличием граничных условий резонатора от идеальных, конечностью диаметра стержня и релаксационными свойствами материала - отожженной меди. Резонансная частота $F_{1} \cong 2204 \mathrm{~Hz}$ первой моды соответствует тому, что фазовая скорость $C_{0}$ НЧ продольной волны в стержне равна $3.8 \cdot 10^{3} \mathrm{~m} / \mathrm{s}$.

Измерения эффектов АЗВТ проводились при комнатной температуре как на прямом, так и на обратном ходе, т.е. при увеличении и при уменьшении амплитуды возбуждения резонатора. Результаты этих измерений практически совпадали. Погрешности измерения частоты и амплитуды акустических колебаний резонатора составляли соответственно $\pm 5 \cdot 10^{-1} \mathrm{~Hz}$ и $\pm 5 \cdot 10^{-2} \mathrm{~dB}$.

\section{2. Результаты измерений эффектов A3BT}

Здесь приведены результаты экспериментальных исследований эффектов АЗВТ (нелинейных потерь и сдвига резонансных частот) в резонаторе для его первых трех продольных мод $(p=1,2,3$,$) . Пьезокерамическим$ излучателем 2 в резонаторе 1 возбуждались НЧ акустические колебания на частоте $F=F_{p}\left(\varepsilon_{m}\right)$, близкой к частоте $F_{p}$ одной из первых трех продольных мод, и измерялись зависимости нелинейных коэффициентов затухания $\mu_{n l, p}\left(\varepsilon_{m}\right)$ и нелинейных сдвигов резонансных частот $\Delta F_{n l, p}\left(\varepsilon_{m}\right)=F_{p}\left(\varepsilon_{m}\right)-F_{p}<0$ от амплитуды деформации $\varepsilon_{m}$ стержня в резонансе.

На рис. 3 приведены зависимости амплитуд деформации $\varepsilon_{m}$ от амплитуды $V_{0}$ гармонического (электрического) напряжения на излучателе накачки $2\left(V_{0} \propto A_{0}\right.$, $A_{0}$ - амплитуда колебаний излучателя 2). Из этого рисунка следует, что при малых амплитудах деформации $\left(\varepsilon_{m} \leq 2 \cdot 10^{-7}\right)$ имеют место линейные зависимости $\varepsilon_{m}$ от $V_{0}$ (т.е. $\left.\varepsilon_{m} \propto V_{0}\right)$, затем при $2 \cdot 10^{-7}<\varepsilon_{m}<4 \cdot 10^{-6}$ зависимости становятся нелинейными, причем $\varepsilon_{m}$ растет 


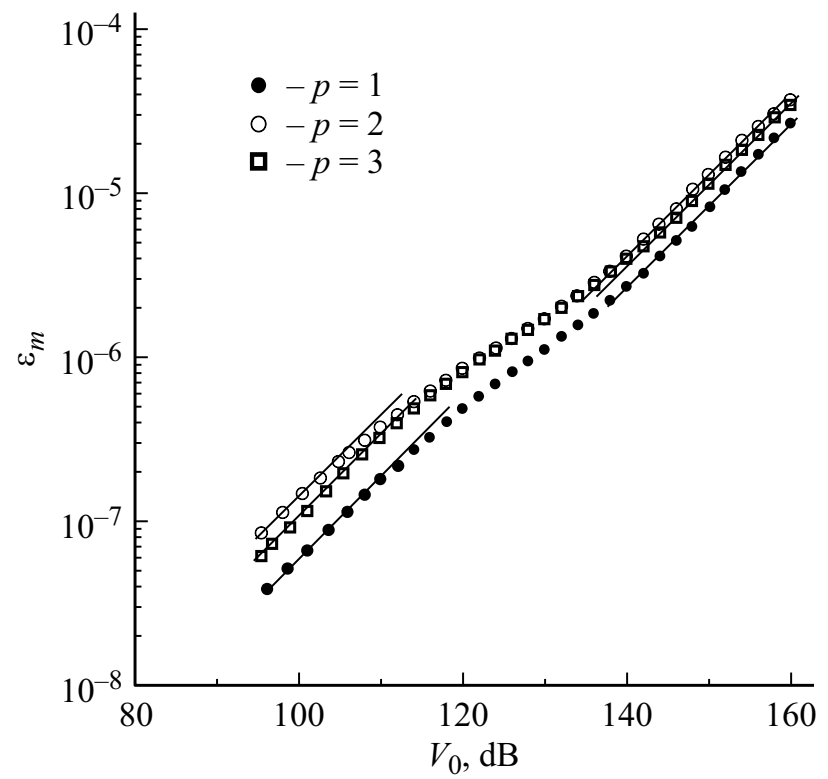

Рис. 3. Зависимости амплитуды $\varepsilon_{m}$ (в резонансе) от амплитуды электрического напряжения на излучателе накачки (в $\mathrm{dB}$ относительно $1 \mu \mathrm{V}$ ). Прямые линии соответствуют зависимостям $\varepsilon_{m} \propto V_{0}$.

медленнее, чем $V_{0}$, а при $\varepsilon_{m} \geq 4 \cdot 10^{-6}-$ опять наблюдаются линейные зависимости $\varepsilon_{m}$ от $V_{0}$. Такие зависимости $\varepsilon_{m}$ от $V_{0}$ свидетельствуют о наличии нелинейных потерь и их насыщении при $\varepsilon_{m} \geq 4 \cdot 10^{-6}$. Из экспериментально установленных зависимостей $\varepsilon_{m}=\varepsilon_{m}\left(V_{0}\right)$ (рис. 2) определим соответствующие им зависимости $\mu_{n l, p}\left(\varepsilon_{m}\right)$ от $\varepsilon_{m}$ для каждой моды резонатора:

$$
\mu_{n l, p}\left(\varepsilon_{m}\right)=\mu_{p}\left(\frac{\varepsilon_{m, 1}}{\varepsilon_{m}} \frac{V_{0}}{V_{0.1}}-1\right),
$$

где $\varepsilon_{m, 1}$ и $V_{0,1}$ - начальные экспериментальные значения амплитуды деформации стержня и амплитуды напряжения на излучателе, когда нелинейные потери пренебрежимо малы и $\varepsilon_{m} \propto V_{0}$, а $\varepsilon_{m}$ и $V_{0}$ - текущие значения амплитуды деформации и амплитуды напряжения на излучателе, когда нелинейные потери проявляются вполне заметно $\varepsilon_{m}>\varepsilon_{m, 1}, \quad V_{0}>V_{0.1}, \mu_{p}=Q_{p}^{-1}$ - линейные потери в резонаторе. На рис. 4 показаны (в малом диапазоне амплитуд $\left.\varepsilon_{m}<2.5 \cdot 10^{-6}\right)$ зависимости $\mu_{n l, p}\left(\varepsilon_{m}\right)$ и $\left|\Delta F_{n l, p}\left(\varepsilon_{m}\right)\right| / F_{p}$ от $\varepsilon_{m}$. Из рис. 4 видно, что при относительно малых амплитудах $\varepsilon_{m}\left(\varepsilon_{m}^{*}<\varepsilon_{m}<1.5 \cdot 10^{-6}\right)$ имеют место следующие соотношения: $\mu_{n l, p}\left(\varepsilon_{m}\right) \approx$ $\approx k_{1}\left(\varepsilon_{m}-\varepsilon_{m}^{*}\right), \quad\left|\Delta F_{n l, p}\left(\varepsilon_{m}\right)\right| / F_{p} \approx k_{2}\left(\varepsilon_{m}-\varepsilon_{m}^{*}\right), \quad$ где $k_{1,2}=$ const $>0, \quad \varepsilon_{m}^{*} \approx 2 \cdot 10^{-7}$. Таким образом, из рис. 4 следует, что эффекты АЗВТ проявляются при $\varepsilon_{m}>\varepsilon_{m}^{*} \approx 2 \cdot 10^{-7}$, т. е. имеют пороговый характер по амплитуде $\varepsilon_{m}$. Из рисунка также следует, что с ростом частоты $F \approx F_{p}$ возбуждения резонатора (при $\left.\varepsilon_{m}=\mathrm{const}>\varepsilon_{m}^{*}\right)$ значения $\mu_{n l, p}\left(\varepsilon_{m}\right)$ и $\left|\Delta F_{n l, p}\left(\varepsilon_{m}\right)\right| / F_{p}$ заметно уменьшаются. Это свидетельствует об уменьшении гистерезисной нелинейности отожженной меди при увеличении частоты деформирования $F \approx F_{p}$.
На рис. 5 показаны зависимости $\mu_{n l, p}\left(\varepsilon_{m}\right)$ и $\left|\Delta F_{n l, p}\left(\varepsilon_{m}\right)\right| / F_{p}$ от $\varepsilon_{m}$ (во всем измеряемом диапазоне амплитуд $\left.\varepsilon_{m}\right)$. Из рис. 5, $a$ видно, что при $\varepsilon_{m} \geq \varepsilon_{m, t h} \approx 4 \cdot 10^{-6}$ имеет место насыщение нелинейных потерь: $\mu_{n l, p}\left(\varepsilon_{m}\right)$ не зависит от $\varepsilon_{m}$. Из рис. 5, $b$ следует, что при $\varepsilon_{m}>\varepsilon_{m, t h} \approx 4 \cdot 10^{-6}$ в зависимостях $\left|\Delta F_{n l, p}\left(\varepsilon_{m}\right)\right| / F_{p}$ от $\varepsilon_{m}$ только проявляется тенденция к насыщению, при этом $\left|\Delta F_{n l, p}\left(\varepsilon_{m}\right)\right| / F_{p} \propto \varepsilon_{m}^{1 / 2}$. (В аналогичном резонаторе из той же меди, но неотожженной, насыщения эффектов АЗВТ не наблюдалось, при этом имели место близкие к линейным зависимости $\mu_{n l, p}\left(\varepsilon_{m}\right)$ и $\Delta F_{n l, p}\left(\varepsilon_{m}\right)$ от $\left.\varepsilon_{m}.\right)$
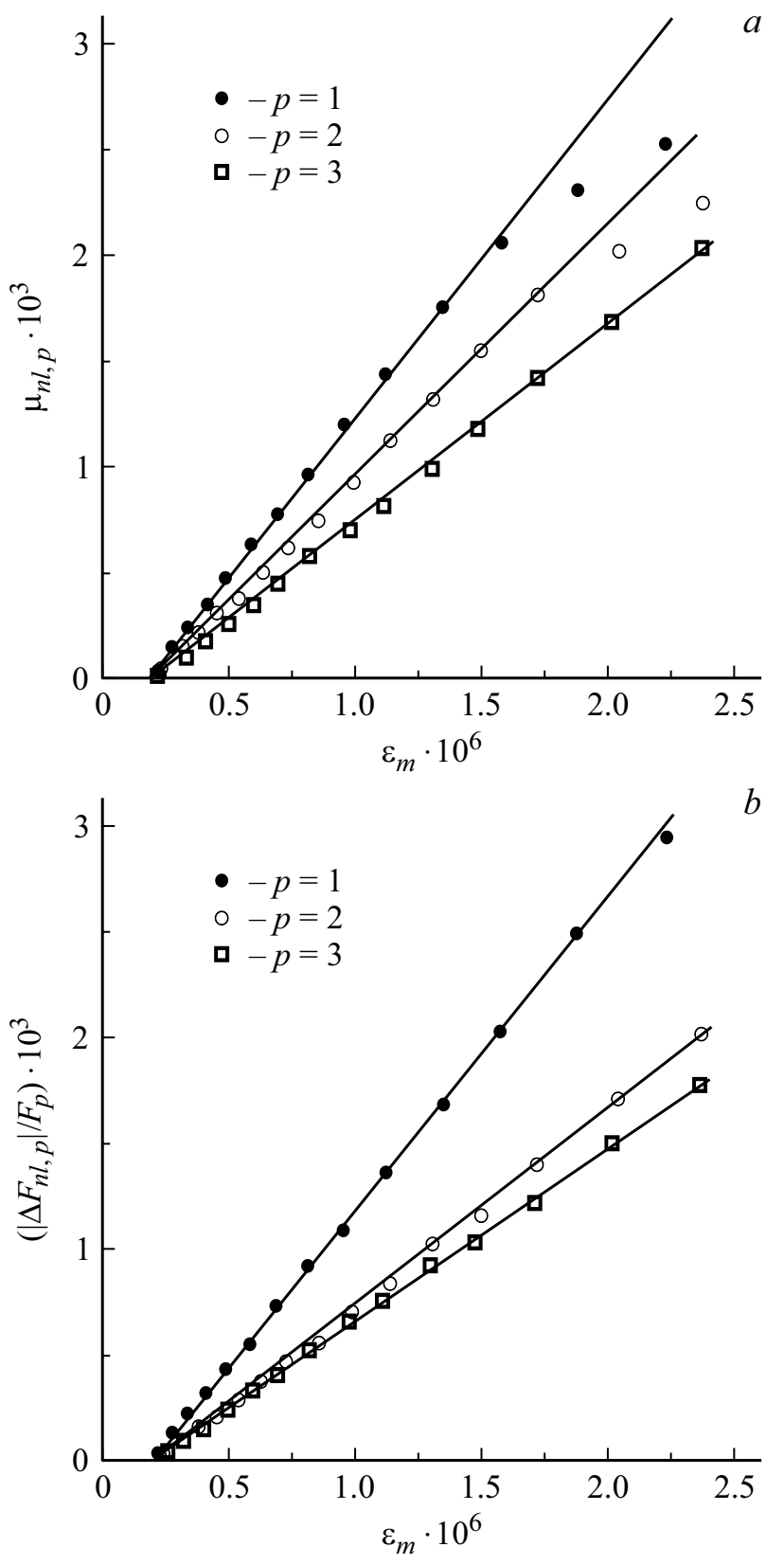

Рис. 4. Зависимости $\mu_{n l, p}\left(\varepsilon_{m}\right) \quad(a)$ и $\left|\Delta F_{n l, p}\left(\varepsilon_{m}\right)\right| / F_{p} \quad(b)$ от $\varepsilon_{m}$. Прямые линии соответствуют зависимостям $\mu_{n l, p}\left(\varepsilon_{m}\right) \propto\left(\varepsilon_{m}-\varepsilon_{m}^{*}\right) ;\left|\Delta F_{n l, p}\left(\varepsilon_{m}\right)\right| / F_{p} \propto\left(\varepsilon_{m}-\varepsilon_{m}^{*}\right)$. 

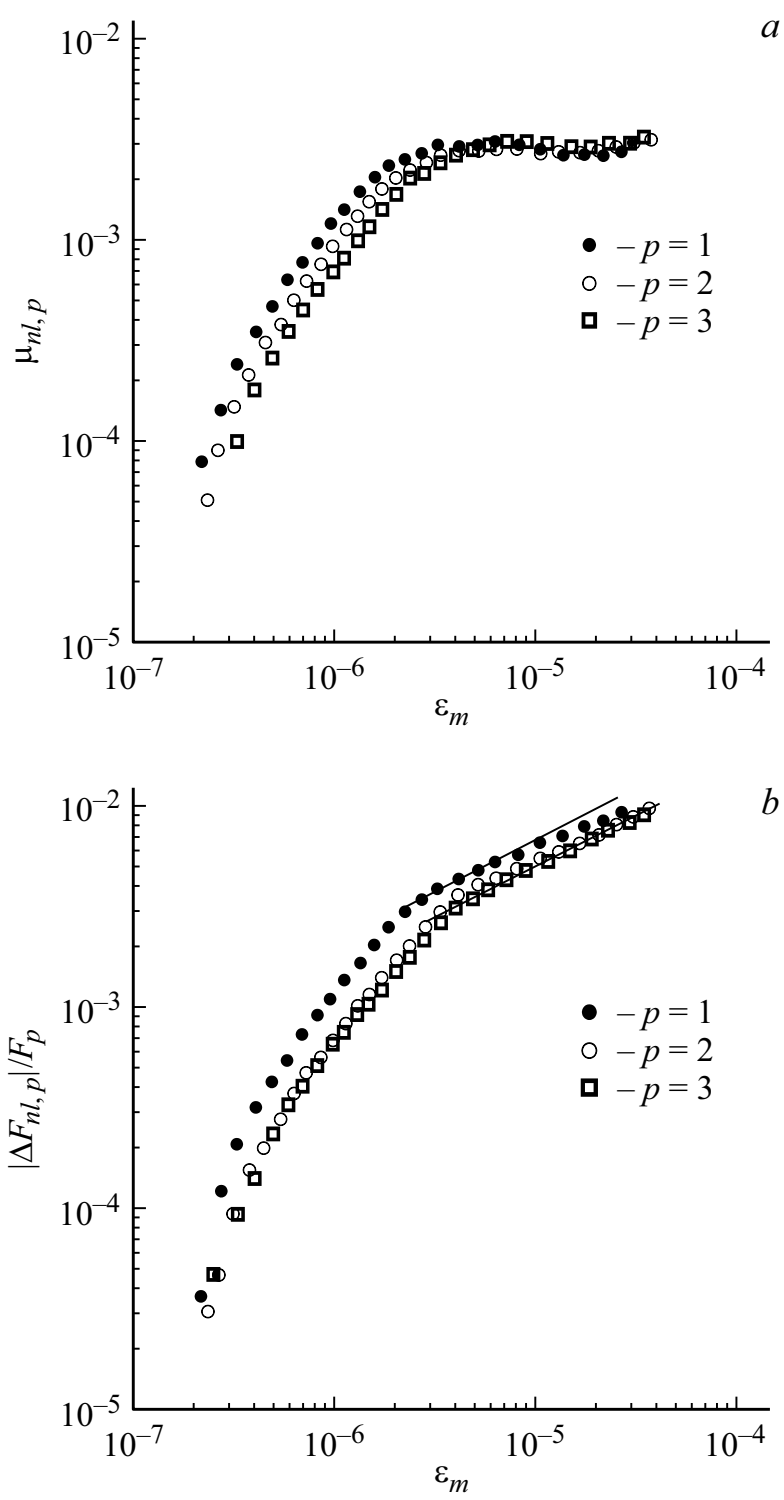

Рис. 5. Зависимости $\mu_{n l, p}(a)$ и $\left|\Delta F_{n l, p}\right| / F_{p}(b)$ от $\varepsilon_{m}$. Прямые линии соответствуют зависимостям $\left|\Delta F_{n l, p}\right| / F_{p} \propto \varepsilon_{m}^{1 / 2}$.

Далее, при аналитическом описании эффектов АЗВТ мы будем рассматривать область достаточно больших амплитуд деформаций $\left(\varepsilon_{m} \gg \varepsilon_{m}^{*} \approx 2 \cdot 10^{-7}\right)$, для которых в начале наблюдаются близкие к линейным зависимости $\mu_{n l, p}\left(\varepsilon_{m}\right)$ и $\Delta F_{n l, p}\left(\varepsilon_{m}\right) / F_{p}$ от $\varepsilon_{m}$, а при $\varepsilon_{m} \geq \varepsilon_{m, t h} \approx 4 \cdot 10^{-6}$ в этих зависимостях имеет место насыщение для $\mu_{n l, p}\left(\varepsilon_{m}\right)$ и проявляется тенденция к насыщению для $\Delta F_{n l, p}\left(\varepsilon_{m}\right) / F_{p}$, при которой $\left|\Delta F_{n l, p}\left(\varepsilon_{m}\right)\right| / F_{p} \propto \varepsilon_{m}^{1 / 2}$.

\section{3. Аналитическое описание эффектов АЗВТ в резонаторе}

Для решения волновых задач, связанных с описанием экспериментально-наблюдаемых нелинейных эффектов в микронеодородных средах, необходимы:

1) знание нелинейных уравнений состояния этих сред;
2) получение корректных аналитических решений соответствующих нелинейных волновых уравнений;

3) их сравнение с результатами экспериментальных исследований нелинейных эффектов в этих средах.

Часто для описания гистерезисных микронеоднородных сред применяются феноменологические уравнения состояния. Такие уравнения, по существу, постулируются на основе анализа результатов экспериментальных исследований нелинейных эффектов, поэтому они, как правило, адекватно описывают эти результаты.

Аналитическое описание эффектов АЗВТ проведем в рамках реологической модели и уравнения состояния микронеоднородной среды $[2,6]$, содержащей нелинейные (гистерезисные) вязко-упругие дефекты. Модель (рис. 6) состоит из одномерной цепочки масс $m$, линейно-упругих жестких элементов - пружинок (с коэффициентами упругости $\kappa$ ) и относительно мягких гистерезисных вязко-упругих дефектов с линейной упругостью $\kappa_{1}=\xi \kappa \ll \kappa$, где $\xi-$ относительная упругость дефектов, $\xi \ll 1$. В этой модели однородные участки цепочки, состоящие из масс и жестких упругих элементов, соответствуют областям бездефектного идеально упругого твердого тела - монокристалла [37], а части цепочки, состоящие из нелинейных вязко-упругих элементов, соответствуют относительно мягким гистерезисным дефектам. (Следует отметить, что такая же реологическая модель гистерезисного вязко-упругого дефекта была рассмотрена в работе [28].) Предполагается, что жесткие и мягкие элементы цепочки имеют одинаковую длину $l \ll \Lambda(\Lambda-$ длина волны), при этом число всех элементов на длине $L$ равно $v(L=v l \gg l)$, а количество дефектов $v_{d}=v N$, где $N=v_{d} / v-$ их концентрация. В соответствии с моделью, при $\Omega \ll(\xi \kappa / m)^{1 / 2}(\Omega-$ частота деформирования), уравнение состояния одного такого дефекта имеет вид

$$
\sigma(\xi)=\xi E[\xi-f(\xi)]+\alpha \dot{\xi},
$$

где $\sigma$ и $\xi-$ напряжение и деформация, $E=\kappa l-$ модуль упругости жестких элементов, $f(\xi)-$ нелинейная (в данном случае, гистерезисная) функция $\xi$, $\alpha-$ коэффициент линейной диссипации демпфера, $|f(\xi)| \ll|\xi| \ll 1$.

Здесь мы будем полагать, что нелинейные свойства дефектов не одинаковы, а так же как их линейные характеристики - относительные упругости $\xi$ и релак-

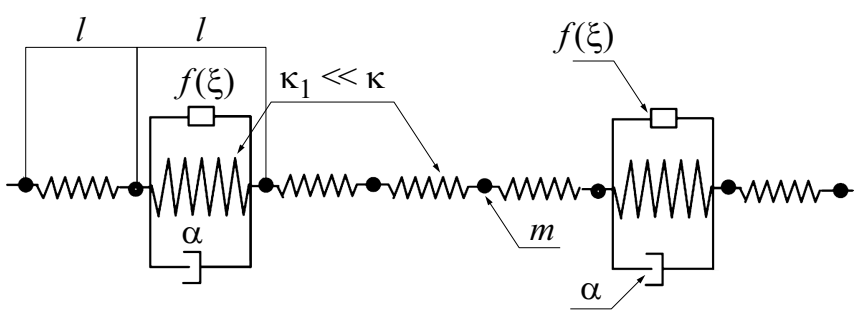

Рис. 6. Реологическая модель микронеоднородной нелинейной среды с релаксацией. 
сационные частоты $W=\xi E / \alpha$, имеют некоторое распределение по параметрам нелинейности, а именно по параметрам $a, b$ и $\gamma_{0}$, отвечающим за гистерезисные потери, дефект модуля упругости и их насыщение. Кроме того, мы также учтем линейные потери бездефектной части твердого тела. В результате уравнения состояния микронеоднородной среды будет иметь вид

$$
\begin{aligned}
& \sigma(\varepsilon)=E\left[\varepsilon-\int_{0}^{\infty} \int_{0}^{1} R(\varepsilon) N(\xi, W) d \xi d W\right. \\
& \left.-\int_{0}^{\infty} \int_{0} \int_{a} \int_{b} \int_{\gamma_{0}} \zeta R\{f[R(\varepsilon)]\} N\left(\xi, W, a, b, \gamma_{0}\right) d \xi d W d a d b d \gamma_{0}\right]
\end{aligned}
$$$$
+\eta \rho \varepsilon
$$

где $\sigma$ и $\varepsilon-$ продольные напряжение и деформация, $R(\varepsilon)=\frac{W}{\xi} \int_{-\infty}^{t} \varepsilon(\tau) \exp [-W(t-\tau)] d \tau, N\left(\xi, W, a, b, \gamma_{0}\right)-$ функция распределения дефектов по параметрам $\xi, W$, $a, b$ и $\gamma_{0}, \iint_{\zeta} \iint_{b} \int_{\gamma_{0}} N\left(\xi, W, a, b, \gamma_{0}\right) d \xi d W d a d b d \gamma_{0}=N_{0}$, $\int_{\xi} \int_{a} \int_{b} \gamma_{0}$

$0<\xi \ll 1,0<W<\infty, \eta-$ кинематический коэффициент линейных потерь, $\rho$ - плотность,

$$
\begin{aligned}
& \left|\int_{0}^{\infty} \int_{0} \int_{a} \int_{b} \int_{\gamma_{0}} \xi R\{f[R(\varepsilon)]\} N\left(\xi, W, a, b, \gamma_{0}\right) d \xi d W d a d b d \gamma_{0}\right| \\
& \left.\ll \mid \int_{0}^{\infty} \int_{0}^{1} R(\varepsilon)\right] N(\xi, W) d \xi d W|\ll| \varepsilon \mid \ll 1 .
\end{aligned}
$$

Из уравнения (3) следует, что нелинейные свойства дефектов (и их распределение по параметрам $a, b$ и $\gamma_{0}$ ) не влияют на линейные свойства микронеоднородной среды, но линейные свойства дефектов влияют на ее нелинейность.

В НЧ диапазоне $(\Omega \ll W)$ уравнение состояния (3) сводится к простому уравнению с безынерционной (частотно-независимой) нелинейностью и линейной диссипацией:

$$
\sigma(\varepsilon)=E_{0}[\varepsilon-\mu f(\varepsilon)]+\eta_{0} \dot{\varepsilon},
$$

где

$$
\begin{gathered}
E_{0}=E\left[1-\int_{0}^{\infty} \int_{0}^{1} \frac{N(\xi, W)}{\xi} d \xi d W\right] \\
\eta_{o}=E \int_{0}^{\infty} \int_{0}^{1} \frac{N(\xi, W)}{\zeta W} d \xi d W
\end{gathered}
$$

$$
\begin{gathered}
\mu=\left[\int_{0}^{\infty} \int_{0}^{1} \int_{a} \int_{b} \int_{\gamma_{0}} \frac{N\left(\xi, W, a, b, \gamma_{0}\right)}{\xi^{2}} d \xi d W d a d b d \gamma_{0}\right] \\
\times\left[1-\int_{0}^{\infty} \int_{0}^{1} \frac{N(\xi, W)}{\zeta} d \xi d W\right]^{-1}, \\
\mu|f(\varepsilon)| \ll|\varepsilon|, \quad \eta_{0}|\dot{\varepsilon}| \ll E_{0}|\varepsilon| .
\end{gathered}
$$

В соответствии с результатами измерений, а именно с установленными амплитудными зависимостями эффектов АЗВТ, будем полагать, что нелинейная функция $f(\xi)$, описывающая гистерезисные свойства дефектов, имеет вид [6]

$$
\begin{aligned}
f(\xi)= & \frac{1}{2\left(1+\gamma_{0}|\xi|\right)} \\
& \times \begin{cases}\gamma_{1} \xi^{2}, & \xi \geq 0, \dot{\xi}>0 \\
-\gamma_{2} \xi^{2}+\left(\gamma_{1}+\gamma_{2}\right) \xi_{m} \xi, & \xi \geq 0, \dot{\xi}<0 \\
-\gamma_{3} \xi^{2}, & \xi \leq 0, \dot{\xi}<0 \\
\gamma_{4} \xi^{2}+\left(\gamma_{3}+\gamma_{4}\right) \xi_{m} \xi, & \xi \leq 0, \dot{\xi}>0\end{cases}
\end{aligned}
$$

где $\xi, \xi_{m}$ и $\xi-$ деформация, амплитуда деформации и скорость деформации дефектов, $\gamma_{0}$ - параметр насыщения, $\gamma_{1-4}$ - параметры гистерезисной нелинейности, $\gamma_{0} \geq 0, \gamma_{1,3}+\gamma_{2,4} \geq 0,\left|\gamma_{1-4}\right| \xi_{m} \ll 1,\left|\gamma_{1-4}\right| \gg 1$.

Гистерезис (5) аналогичен безынерционному дислокационному гистерезису Гранато-Люкке [9-14]; он также состоит из четырех ветвей, проходящих через нулевую точку: $f(\xi=0)=0$. В результате в квазистатике $(\Omega \ll W$, или при $\alpha=0$ и $\eta=0)$ все четыре ветви гистерезисного уравнения состояния (4), (5) также будут проходить через нулевую точку: $\sigma(\varepsilon=0)=0$. Отличия динамического гистерезиса (3), (5) от безынерционного гистерезиса Гранато-Люкке связаны, во-первых, с квадратичными, а не экспоненциальными и линейными, зависимостями $\sigma=\sigma(\varepsilon)$ для ветвей нагрузки $(\varepsilon \geq 0, \dot{\varepsilon}>0$ и $\varepsilon \leq 0, \dot{\varepsilon}<0)$ и разгрузки $(\varepsilon \geq 0, \dot{\varepsilon}<0$ и $\varepsilon \leq 0, \dot{\varepsilon}>0)$ при $\gamma_{0} \varepsilon_{m} \ll \xi$, во-вторых, с насыщением эффектов АЗВТ при $\gamma_{0} \varepsilon_{m} \gg \zeta$ и в-третьих, с частотной зависимостью эффектов АЗВТ.

В теории Гранато-Люкке гистерезис в зависимости $\sigma=\sigma(\varepsilon)$ связывается с поведением дислокаций под действием знакопеременного напряжения, а именно с последовательным и лавинообразным (по существу, мгновенным) отрывом сегментов дислокаций от примесных атомов - при нагрузке $(\varepsilon \geq 0, \dot{\varepsilon}>0$ и $\varepsilon \leq 0, \dot{\varepsilon}<0)$ и одновременным закреплением на них же - при разгрузке $(\varepsilon \geq 0, \dot{\varepsilon}<0$ и $\varepsilon \leq 0, \dot{\varepsilon}>0)$. Отрыв сегментов дислокаций от примесных атомов происходит пороговым образом (при $\varepsilon_{m}>\varepsilon_{m}^{*}$ ), поэтому эффекты АЗВТ пороговые, что и отмечалось при анализе рис. 4. В несколько видоизмененных теориях дислокационного поглощения $[38,39]$ предполагается, что движение сегментов дислокаций, оторвавшихся от примесных атомов, ограничивается не только их линейным натяжением, 
но и полем упругих напряжений соседних примесных атомов. Отрывающиеся от примесных атомов сегменты дислокации перезакрепляются на соседних примесных атомах — этот механизм ограничивает увеличение длины дислокации и рост площади петель гистерезисной зависимости $\sigma=\sigma(\varepsilon)$. Такое движение дислокаций приводит в начале к линейной зависимости эффектов АЗВТ от амплитуды деформации $\varepsilon_{m}$, а затем - к их насыщению, при этом разгрузочные ветви кривой $\sigma=\sigma(\varepsilon)$, также как и нагрузочные, становятся нелинейными.

Отметим, что в действительности отрыв сегментов дислокаций от примесных атомов (и их последующее перезакрепление на соседних) не является мгновенным (как в теории Гранато-Люкке), а составляет конечное время $T$, что определяет частоту релаксации $W=2 \pi / T$ дислокации и соответственно уменьшение гистерезисной нелинейности поликристалла с ростом частоты упругой волны при (рис. 4).

Необходимо, однако, заметить, что установленные амплитудные зависимости эффектов АЗВТ в отожженной поликристаллической меди можно описывать не только гистерезисной функцией (5), качественно соответствующей упругому гистерезису Гранато-Люкке, но и гистерезисной функцией, соответствующей модифицированному [6] микропластическому гистерезису Давиденкова [7]:

$$
\begin{aligned}
f(\xi)= & \frac{\beta \xi_{m} \xi}{1+\gamma_{0} \xi_{m}}+\frac{1}{2\left(1+\gamma_{0} \xi_{m}\right)} \\
& \times \begin{cases}\beta_{1} \xi^{2}-\frac{\beta_{1}+\beta_{2}}{2} \xi_{m}^{2}, & \dot{\xi}>0 ; \\
-\beta_{2} \xi^{2}+\frac{\beta_{1}+\beta_{2}}{2} \xi_{m}^{2}, & \dot{\xi}<0,\end{cases}
\end{aligned}
$$

где $\gamma_{0}, \beta, \beta_{1,2}$ - параметры гистерезисной нелинейности, $\gamma_{0} \geq 0, \beta \xi_{m} \ll 1, \beta_{1,2} \xi_{m} \ll 1,|\beta| \gg 1,\left|\beta_{1,2}\right| \gg 1$, $\beta_{1}+\beta_{2} \geq 0$. Такой гистерезис состоит из двух ветвей положительной $(\dot{\xi}>0)$ и отрицательной $(\dot{\xi}<0)$, непрерывно переходящих друг в друга при $\xi= \pm \xi_{m}$, и не проходящих через нулевую точку:

$$
f(\xi=0)=\mp \frac{\left(\beta_{1}+\beta_{2}\right) \xi_{m}^{2}}{4\left(1+\gamma_{0} \xi_{m}\right)} \neq 0 .
$$

Здесь гистерезисное поведение кристалла связывается с его необратимой микропластической деформацией [7], а за насыщение эффектов АЗВТ отвечает множитель $\left(1+\gamma_{0} \xi_{m}\right)^{-1}$.

Отметим, что для описания эффектов АЗВТ в отожженной меди выбор гистерезисной функции $f(\xi)$ из двух возможных вариантов (5), (6) определялся из следующих соображений: если $f(\xi)$ определяется выражением (5), то в квазистатике - $\sigma(\varepsilon=0)=0$, а если выражением (6), - то $\sigma(\varepsilon=0) \neq 0$ и $\varepsilon(\sigma=0) \neq 0$. Таким образом, в первом случае напряжение и деформация среды - упругие, а во втором - имеет место остаточное напряжение $\sigma(\varepsilon=0) \neq 0$ и пластическая деформация $\varepsilon(\sigma=0) \neq 0$. Наличие последних привело бы к тому, что и при ненулевых деформациях $\left(\varepsilon_{m} \geq \varepsilon_{m, t h} \approx 4 \cdot 10^{-6}\right)$ также бы имели место и остаточное напряжения и пластическая деформация, при этом наблюдался бы рост и нелинейных потерь и нелинейных сдвигов резонансных частот. Результаты же измерений показали, что при $\varepsilon_{m} \geq \varepsilon_{m, t h} \approx 4 \cdot 10^{-6}$ имеет место только рост нелинейного сдвига резонансной частоты, а это соответствует тому, что при $\varepsilon_{m} \geq \varepsilon_{m, t h} \approx 4 \cdot 10^{-6}$ напряжения и деформации - упругие, и, следовательно, имеет место гистерезис (5).

Подставляя уравнение состояния (3) в уравнение движения $\rho U_{m}=\sigma_{x}(\varepsilon)$ [4], получим волновое уравнение для продольного (вдоль оси $x$ ) смещения $U=U(x, t)$ в резонаторе:

$$
\begin{aligned}
U_{t t} & \left.-C^{2}\left[U_{x x}-\int_{0}^{\infty} \int_{0}^{1} R_{x}(\varepsilon)\right] N(\xi, W) d \xi d W\right] \\
& -\eta U_{x x t}=-C^{2} \int_{0}^{\infty} \int_{0}^{1} \int_{a} \int_{b} \int_{\gamma_{0}} \xi R_{x}\{f[R(\varepsilon)]\} \\
& \times N\left(\xi, W, a, b, \gamma_{0}\right) d \xi d W d a d b d \gamma_{0},
\end{aligned}
$$

где $\varepsilon=U_{x}(x, t), C=(E / \rho)^{1 / 2}-$ скорость продольной волны в среде без дефектов. Для резонатора с жесткой $(x=0)$ и мягкой $(x=L)$ границами граничные условия имеют вид $U(x=0, t)=A_{0} \sin \Omega t, U_{x}(x=L, t)=0$, где $\Omega=2 \pi F$.

Резонансная кривая для такого резонатора имеет вид

$$
\begin{gathered}
\varepsilon_{m}=\frac{A_{0} \Omega_{p} / L}{\left\{\left[\delta_{p}-\delta_{n l, p}\left(\varepsilon_{m}\right)\right]^{2}+\left[\mu_{p}+\mu_{n l, p}\left(\varepsilon_{m}\right)\right]^{2} \Omega_{p}^{2}\right\}^{1 / 2}}, \\
\mu_{p}=Q_{p}^{-1}=\frac{\eta \Omega_{p}}{2 C_{0}^{2}}+\frac{\Omega_{p}}{2} \int_{0}^{\infty} \int_{0}^{1} \frac{N(\xi, W) d \xi d W}{\xi W\left[1+\left(\Omega_{p} / W\right)^{2}\right]}, \\
\Omega_{p}=\Omega_{p}^{*}\left[1-\int_{0}^{\infty} \int_{0}^{1} \frac{N(\xi, W) d \xi d W}{2 \xi\left[1+\left(\Omega_{p} / W\right)^{2}\right]}\right] \approx \Omega_{p}^{*}, \\
\int_{0}^{\infty} \int_{0}^{1} \frac{N(\xi, W) d \xi d W}{2 \xi\left[1+\left(\Omega_{p} / W\right)^{2}\right]} \ll 1,
\end{gathered}
$$

где $\Omega_{p}=2 \pi F_{p}, \Omega_{p}^{*}=2 \pi F_{p}^{*}=(2 p-1) \pi C / 2 L-$ резонансные частоты идеального (без дефектов) резонатоpa, $\delta_{p}=\Omega-\Omega_{p},\left|\delta_{p}\right| \ll \Omega_{p} / p, \delta_{n l, p}\left(\varepsilon_{m}\right)=2 \pi \Delta F_{n l, p}\left(\varepsilon_{m}\right)$, а нелинейные потери $\mu_{n l, p}\left(\varepsilon_{m}\right)$ и относительный сдвиг резонансной частоты $\delta_{n l, p}\left(\varepsilon_{m}\right) / \Omega_{p}$ определяются нижеследующими выражениями. (Формула (1) следует из выражения (7) при $\delta_{p}=\delta_{n l, p}\left(\varepsilon_{m},\right)$.)

В малоамплитудном режиме $\left(\gamma_{0} \varepsilon_{m} \ll \zeta\right.$, $\left.\varepsilon_{m} \ll\left|a_{\text {eff }}\left(\Omega_{p}\right) / c_{\text {eff }}\left(\Omega_{p}\right)\right|, \quad \varepsilon_{m} \ll\left|b_{\text {eff }}\left(\Omega_{p}\right) / d_{\text {eff }}\left(\Omega_{p}\right)\right|\right)$ имеем

$$
\mu_{n l, p}\left(\varepsilon_{m}\right)=\left[a_{\mathrm{eff}}\left(\Omega_{p}\right)-c_{\mathrm{eff}}\left(\Omega_{p}\right) \varepsilon_{m}\right] \varepsilon_{m} \approx a_{\mathrm{eff}}\left(\Omega_{p}\right) \varepsilon_{m},
$$




$$
\begin{aligned}
\frac{\delta_{n l, p}\left(\varepsilon_{m}\right)}{\Omega_{p}}= & -\left[b_{\mathrm{eff}}\left(\Omega_{p}\right)-d_{\mathrm{eff}}\left(\Omega_{p}\right) \varepsilon_{m}\right] \varepsilon_{m} \approx \\
& -b_{\mathrm{eff}}\left(\Omega_{p}\right) \varepsilon_{m},
\end{aligned}
$$

где

$$
\begin{aligned}
& a_{\mathrm{eff}}\left(\Omega_{p}\right)= \\
& =\int_{0}^{\infty} \int_{0}^{1} \int_{a} \int_{b} \int_{\gamma_{0}} \frac{\begin{array}{c}
\left\{a\left[1-\left(\Omega_{p} / W\right)^{2}\right]+2 b\left(\Omega_{p} / W\right)\right\} \times \\
\zeta^{2}\left[1+\left(\Omega_{p} / W\right)^{2}\right]^{5 / 2}
\end{array}}{b_{\mathrm{eff}}\left(\Omega_{p}\right)=} \\
& =\int_{0}^{\infty} \int_{0}^{1} \int_{a} \int_{b} \int_{\gamma_{0}} \frac{\left\{b\left[1-\left(\Omega_{p} / W\right)^{2}\right]-2 a\left(\Omega_{p} / W\right)\right\} \times}{\zeta^{2}\left[1+\left(\xi, W, b, \gamma_{0}\right) d \xi d W d a d b d \gamma_{0}\right.} \\
&
\end{aligned}
$$$$
c_{\text {eff }}\left(\Omega_{p}\right)=
$$$$
=\int_{0}^{\infty} \int_{0}^{1} \int_{a} \int_{b} \int_{\gamma_{0}} \frac{\begin{array}{c}
\left\{c\left[1-\left(\Omega_{p} / W\right)^{2}\right]+2 d\left(\Omega_{p} / W\right)\right\} \times \\
\times \gamma_{0} N\left(\xi, W, a, b, \gamma_{0}\right) d \xi d W d a d b d \gamma_{0}
\end{array}}{\xi^{3}\left[1+\left(\Omega_{p} / W\right)^{2}\right]^{3}},
$$$$
d_{\mathrm{eff}}\left(\Omega_{p}\right)=
$$$$
=\int_{0}^{\infty} \int_{0}^{1} \int_{a} \int_{b} \int_{\gamma_{0}} \frac{\begin{array}{c}
\left\{d\left[1-\left(\Omega_{p} / W\right)^{2}\right]-2 c\left(\Omega_{p} / W\right)\right\} \times \\
\times \gamma_{0} N\left(\xi, W, a, b, \gamma_{0}\right) d \zeta d W d a d b d \gamma_{0}
\end{array}}{\xi^{3}\left[1+\left(\Omega_{p} / W\right)^{2}\right]^{3}}
$$$$
a=\frac{2\left(\gamma_{1}+\gamma_{2}+\gamma_{3}+\gamma_{4}\right)}{9 \pi^{2}} \geq 0,
$$$$
b=\frac{\gamma_{1}+\gamma_{2}+\gamma_{3}+\gamma_{4}}{6 \pi}+\frac{4\left(\gamma_{1}-\gamma_{2}+\gamma_{3}-\gamma_{4}\right)}{9 \pi^{2}},
$$$$
c=\frac{\gamma_{1}+\gamma_{2}+\gamma_{3}+\gamma_{4}}{64 \pi},
$$

$$
d=\frac{\gamma_{1}+\gamma_{2}+\gamma_{3}+\gamma_{4}}{8 \pi}+\frac{9\left(\gamma_{1}-\gamma_{2}+\gamma_{3}-\gamma_{4}\right)}{256} .
$$

В режиме насыщения эффектов АЗВТ $\left(\gamma_{0} \varepsilon_{m} \gg \xi\right.$, $\left.\varepsilon_{m} \gg\left|a_{\mathrm{eff}}\left(\Omega_{p}\right) / c_{\mathrm{eff}}\left(\Omega_{p}\right)\right|, \varepsilon_{m} \gg\left|b_{\mathrm{eff}}\left(\Omega_{p}\right) / d_{\mathrm{eff}}\left(\Omega_{p}\right)\right|\right)$ получаем

$$
\begin{aligned}
& \mu_{n l, p}\left(\varepsilon_{m}\right)= \\
& =\int_{0}^{\infty} \int_{0}^{1} \int_{a} \int_{b} \int_{\gamma_{0}} \frac{\begin{array}{c}
\left\{\bar{a}\left[1-\left(\Omega_{p} / W\right)^{2}\right]+2 \bar{b}\left(\Omega_{p} / W\right)\right\} \times \\
2 \xi \gamma_{0}\left[1+\left(\Omega_{p} / W\right)^{2}\right]^{2}
\end{array}}{\frac{\delta_{n l, p}\left(\varepsilon_{m}\right)}{\Omega_{p}}=} \\
& =-\int_{0}^{\infty} \int_{0}^{1} \int_{a} \int_{b} \int_{\gamma_{0}} \frac{\left\{\bar{b}\left[1-\left(\Omega_{p} / W\right)^{2}\right]-2 \bar{a}\left(\Omega_{p} / W\right)\right\}}{2 \xi \gamma_{0}\left[1+\left(\Omega_{p} / W\right)^{2}\right]^{2}}
\end{aligned}
$$

где

$$
\bar{a}=\frac{\gamma_{1}+\gamma_{2}+\gamma_{3}+\gamma_{4}}{4 \pi}=\frac{9 \pi}{8} a,
$$

$$
\begin{aligned}
\bar{b} & =\frac{1}{2 \pi}\left(\gamma_{1}+\gamma_{2}+\gamma_{3}+\gamma_{4}+\frac{\pi\left(\gamma_{1}-\gamma_{2}+\gamma_{3}-\gamma_{4}\right)}{4}\right) \\
& =\frac{9 \pi^{2}}{32}\left(\frac{32-3 \pi^{2}}{4 \pi} a+b\right) .
\end{aligned}
$$

Из выражений (11), (12) видно, что в малоамплитудном режиме (при $2 \cdot 10^{-7} \approx \varepsilon_{m}^{*}<\varepsilon_{m}<1.5 \cdot 10^{-6}$ ) аналитические зависимости $\mu_{n l, p}\left(\varepsilon_{m}\right)$ и $\left|\Delta F_{n l, p}\left(\varepsilon_{m}\right)\right| / F_{p}$ от $\varepsilon_{m}$ соответствуют экспериментально установленным: $\mu_{n l, p}\left(\varepsilon_{m}\right) \propto \varepsilon_{m},\left|\Delta F_{n l, p}\left(\varepsilon_{m}\right)\right| / F_{p} \propto \varepsilon_{m}$. В режиме же насыщения $\left(\varepsilon_{m} \geq \varepsilon_{m, t h} \approx 4 \cdot 10^{-6}\right)$ такое соответствие имеет место только для нелинейных потерь (15), а для нелинейных сдвигов резонансных частот (16) - соответствия нет: результаты измерений свидетельствуют, что $\left|\Delta F_{n l, p}\left(\varepsilon_{m}\right)\right| / F_{p} \propto \varepsilon_{m}^{1 / 2}$, а из выражения (16) следует, что $\left|\Delta F_{n l, p}\left(\varepsilon_{m}\right)\right| / F_{p}$ от $\varepsilon_{m}$ не зависит. В связи с этим для объяснения зависимости $\left|\Delta F_{n l, p}\left(\varepsilon_{m}\right)\right| / F_{p} \propto \varepsilon_{m}^{1 / 2}$, наблюдаемой при $\varepsilon_{m}>\varepsilon_{k m, t h} \approx 4 \cdot 10^{-6}$, в правую часть уравнения состояния (3) следует добавить нелинейное (негистерезисное и безынерционное) слагаемое следующего вида:

$$
\begin{aligned}
\Delta \sigma(\varepsilon) & =-E \cdot\left\{\begin{array}{cc}
v_{1}\left(|\varepsilon|-\varepsilon_{m, t h}\right)^{3 / 2}, & \varepsilon \geq \varepsilon_{m, t h} \\
o, & |\varepsilon| \leq \varepsilon_{m, t h} \\
v_{2}\left(|\varepsilon|-\varepsilon_{m, t h}\right)^{3 / 2}, & \varepsilon \leq-\varepsilon_{m, t h}
\end{array}\right\} \\
& =-E v_{1,2}\left(|\varepsilon|-\varepsilon_{m, t h}\right)^{3 / 2} h\left(|\varepsilon|-\varepsilon_{m, t h}\right)
\end{aligned}
$$

где $v_{1,2}=$ const, $h\left(|\varepsilon|-\varepsilon_{m, t h}\right)-$ функция Хэвисайда.

В этом случае нелинейные потери по-прежнему будут определяться выражением (15), а приращение относительного сдвига резонансной частоты (при $\varepsilon_{m}>\varepsilon_{m, t h} \approx 4 \cdot 10^{-6}$ ) к значению, определяемому выражением $(16)$ и достигаемому при $\varepsilon_{m}=\varepsilon_{m, t h} \approx 4 \cdot 10^{-6}$, будет иметь вид, соответствующий результатам измерений:

$$
\Delta\left(\frac{\Delta F_{n l, p}\left(\varepsilon_{m}\right)}{F_{p}}\right) \approx-\left(\frac{12 \pi}{5 \Gamma^{2}(1 / 4)}\right)^{2}\left(v_{1}-v_{2}\right)\left(\varepsilon_{m}-\varepsilon_{m, t h}\right)^{1 / 2} .
$$

Следует отметить, что зависимость (17) близка к соотношению $\Delta \sigma(\varepsilon) \propto(-\varepsilon)^{3 / 2} h(-\varepsilon)$, характерному для контактного взаимодействия двух упругих твердых тел [4]; она имеет ту же дробную степень, равную $3 / 2$, что соответствует так называемой герцевской нелинейности. Вполне возможно, что и здесь (при $\varepsilon_{m}>\varepsilon_{m, t h} \approx 4 \cdot 10^{-6}$ ) имело место контактное герцевское взаимодействие упругих зерен поликристаллической отожженной меди (рис. $1, b)$. Во всяком случае, такое объяснение вполне можно рассматривать в качестве рабочей гипотезы. 


\section{4. Определение эффективных параметров нелинейности}

Из выражений (11)-(14) следует, что по результатам измерений эффектов АЗВТ можно определить значения эффективных параметров нелинейности $a_{\mathrm{eff}}\left(F_{p}\right)$ и $b_{\text {eff }}\left(F_{p}\right)$, а затем и функцию распределения дефектов $N=N\left(\xi, W, a, b, \gamma_{0}\right)$, отвечающую зависимости параметров $a_{\mathrm{eff}}\left(F_{p}\right)$ и $b_{\mathrm{eff}}\left(F_{p}\right)$ от частоты $F_{p}=\Omega_{p} / 2 \pi$.

В малоамплитудном режиме из сравнения выражений $(11),(12)$ с результатами измерений (рис. 4) находим (при $\varepsilon_{m}=1.5 \cdot 10^{-6} \gg \varepsilon_{m}^{*} \approx 2 \cdot 10^{-7}$ ) значения эффективных параметров $a_{\text {eff }}\left(F_{p}\right)$ и $b_{\text {eff }}\left(F_{p}\right)$ для трех значений $F_{p}$ (рис. 7). Далее подберем функцию распределения дефектов $N=N\left(\xi, W, a, b, \gamma_{0}\right)$, отвечающую зависимостям параметров $a_{\text {eff }}\left(F_{p}\right)$ и $b_{\text {eff }}\left(F_{p}\right)$ от частоты $F_{p}$. В наиболее простом варианте такая функция имеет следующий вид:

$$
\begin{aligned}
& N\left(\xi, W, a, b, \gamma_{0}\right)=N_{0}\left[v \delta\left(\xi-\xi_{1}\right) \delta\left(W-W_{1}\right)\right. \\
& \times \delta\left(a-a_{1}\right) \delta\left(b-b_{1}\right) \delta\left(\gamma_{0}-\gamma_{0,1}\right)+(1-v) \delta\left(\xi-\xi_{2}\right) \\
& \left.\times \delta\left(W-W_{2}\right) \delta\left(a-a_{2}\right) \delta\left(b-b_{2}\right) \delta\left(\gamma_{0}-\gamma_{0,2}\right)\right],
\end{aligned}
$$

где $N_{0}-$ концентрация дефектов, $N_{0}=6.5 \cdot 10^{-6}$, $v=4.6 \cdot 10^{-2}, \xi_{1}=10^{-3}, W_{1}=6 \cdot 10^{4} \mathrm{~s}^{-1}, a_{1}=9 \cdot 10^{2}$, $b_{1}=4 \cdot 10^{3}, \quad \xi_{2}=4 \cdot 10^{-3}, \quad W_{2}=10^{4} \mathrm{~s}^{-1}, \quad a_{2}=2 \cdot 10^{3}$, $b_{2}=1.6 \cdot 10^{3}$, а значения параметров $\gamma_{0,1}$ и $\gamma_{0,2}$ определим ниже, из анализа эффектов АЗВТ в режиме насыщения. (Параметры $a_{\mathrm{eff}}\left(F_{p}\right)$ и $b_{\mathrm{eff}}\left(F_{p}\right)$ не зависят от значений $\gamma_{0,1}$ и $\gamma_{0,2}$.) Из выражения (19) следует, что функция $N=N\left(\xi, W, a, b, \gamma_{0}\right)$ отвечает наличию дефектов, имеющих два различных набора значений

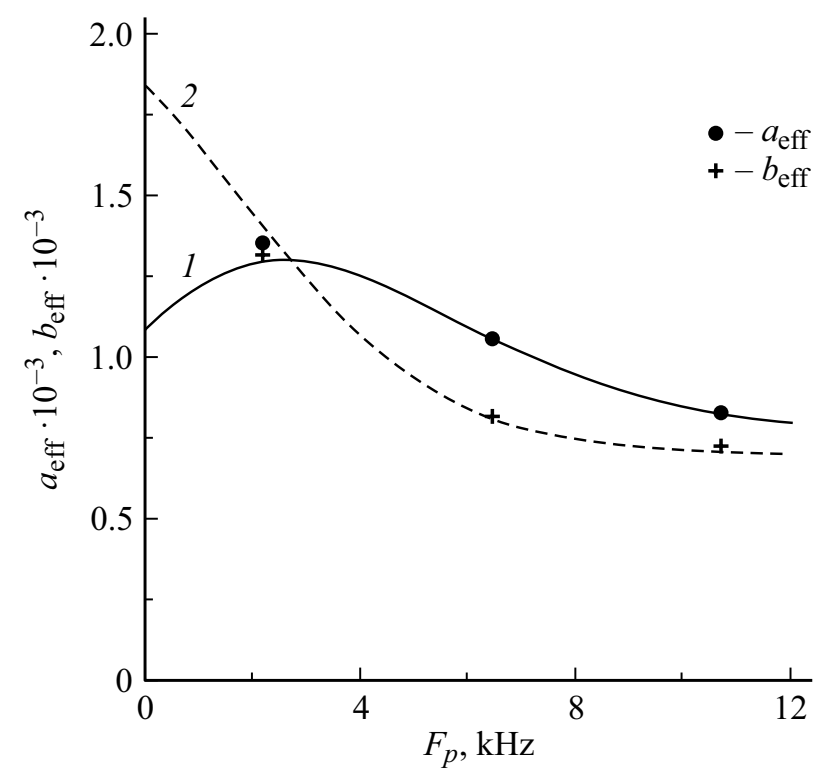

Рис. 7. Зависимости эффективных параметров $a_{\text {eff }}-1$ и $b_{\text {eff }}-2$ от частоты $F_{p} ; \bullet$ и + результаты измерений.

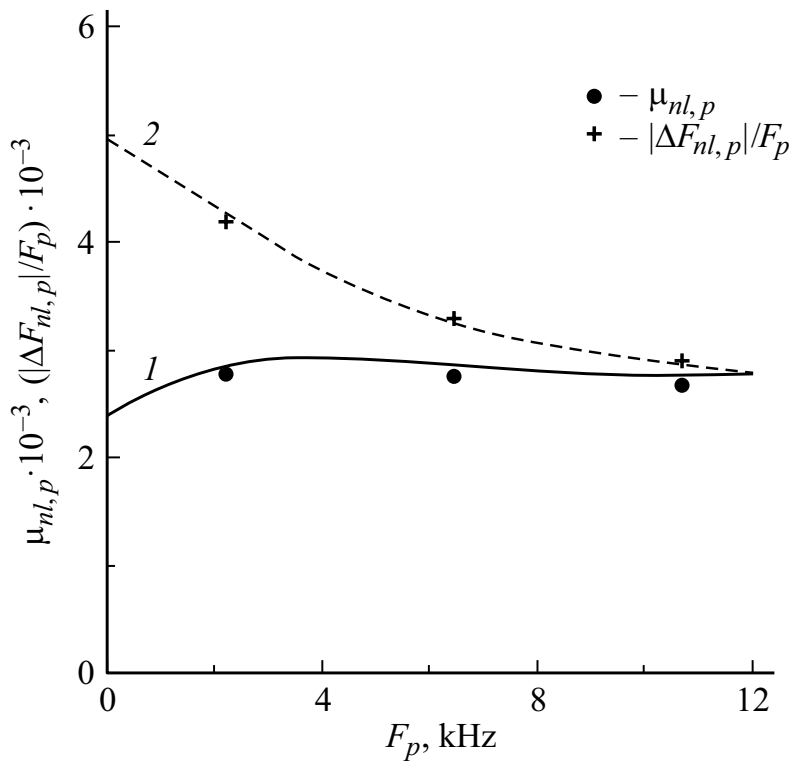

Рис. 8. Зависимости $\mu_{n l, p}\left(\varepsilon_{m}\right)-1$ и $\Delta F_{n l, p}\left(\varepsilon_{m}\right) \mid / F_{p}-2$ от $F_{p}$ в режиме насыщения: • и +- результаты измерений.

параметров $\xi, W, a, b, \gamma_{0}$, при этом их концентрация равна соответственно $v N_{0}$ и $(1-v) N_{0}$.

На рис. 7 отмечены экспериментальные точки и приведены теоретические зависимости (13), (14) для $a_{\mathrm{eff}}\left(F_{p}\right)$ и $b_{\text {eff }}\left(F_{p}\right)$, построенные при заданной функции распределения (19). Из рис. 7 видно хорошее совпадение теоретических зависимостей $a_{\text {eff }}\left(F_{p}\right)$ и $b_{\text {eff }}\left(F_{p}\right)$ с результатами измерений.

Далее в режиме насыщения нелинейных потерь из выражений (15) и (16) при той же функции распределения (19), в которой $\gamma_{0,1}=4.15 \cdot 10^{3}, \gamma_{0,2}=4.6 \cdot 10^{3}$, построим графики зависимостей $\mu_{n l, p}\left(\varepsilon_{m}\right)$ и $\Delta F_{n l, p}\left(\varepsilon_{m}\right) / F_{p}$ от частоты (рис. 8) и отметим на этих графиках экспериментальные точки (из рис. 4), соответствующие $\varepsilon_{m}=4 \cdot 10^{-6}$. (Здесь мы полагаем, что при $\varepsilon_{m}=4 \cdot 10^{-6}$ значение $\Delta F_{n l, p}\left(\varepsilon_{m}\right) / F_{p}$, так же как и $\mu_{n l, p}\left(\varepsilon_{m}\right)$, достигает насыщения.) На рис. 8 видно хорошее совпадение теоретических зависимостей $(15),(16)$ и экспериментальных значений $\mu_{n l, p}\left(\varepsilon_{m}\right)$ и $\Delta F_{n l, p}\left(\varepsilon_{m}\right) / F_{p}$, при этом $\mu_{n l, p}\left(\varepsilon_{m}\right)$ практически не зависит от частоты, а $\Delta F_{n l, p}\left(\varepsilon_{m}\right) / F_{p}$ слабо уменьшается с ее ростом.

Наконец, из сравнения выражения (17) с результатами измерений (рис. $5, b)$, полагая, что $\Delta\left(\frac{\Delta F_{n l, p}\left(\varepsilon_{m}\right)}{F_{p}}\right)$ определяется превышением над $\Delta F_{n l, p}\left(\varepsilon_{m}\right) / F_{p}$ при $\varepsilon_{m}=\varepsilon_{m, t h} \approx 4 \cdot 10^{-6}$, находим $\nu_{1}-v_{2} \approx 3$.

\section{Заключение}

Приведены результаты экспериментальных и теоретических исследований эффектов АЗВТ в стержневом резонаторе из отожженной поликристаллической меди, а именно нелинейных потерь $\mu_{n l, p}\left(\varepsilon_{m}\right)$ и сдвига резонансных частот $\Delta F_{n l, p}\left(\varepsilon_{m}\right)$. Измерения проводились на 
первых трех продольных модах резонатора в диапазоне частот $F_{p}$ от 2 до $11 \mathrm{kHz}$ и в диапазоне амплитуд $\varepsilon_{m}$ деформаций от $4 \cdot 10^{-8}$ до $4 \cdot 10^{-5}$. Анализ экспериментально установленных амплитудно-частотных зависимостей эффектов АЗВТ в отожженной меди показал, что: 1) ее гистерезисная нелинейность носит пороговый характер, что соответствует дислокационной теории Гранато-Люкке, и проявляется при $\varepsilon_{m}>\varepsilon_{m}^{*} \approx 2 \cdot 10^{-7}$; 2) при малых амплитудах $\varepsilon_{m}>\varepsilon_{m}^{*} \quad\left(\varepsilon_{m}^{*} \approx 2 \cdot 10^{-7}\right.$ $\left.<\varepsilon_{m}<1.5 \cdot 10^{-6}\right)$ имеют место следующие амплитудные зависимости: $\mu_{n l, p}\left(\varepsilon_{m}\right) \propto\left(\varepsilon_{m}-\varepsilon_{m}^{*}\right)>0$, $\Delta F_{n l, p}\left(\varepsilon_{m}\right) / F_{p} \propto-\left(\varepsilon_{m}-\varepsilon_{m}^{*}\right)<0$;

$3)$ эффективные параметры $a_{\text {eff }}\left(F_{p}\right)$ и $b_{\text {eff }}\left(F_{p}\right)$ гистерезисной нелинейности, определяющие нелинейные потери $\mu_{n l, p}\left(\varepsilon_{m}\right)$ и сдвиг резонансных частот $\Delta F_{n l, p}\left(\varepsilon_{m}\right)$ в малоамплитудном режиме, являются частотно-зависимыми и с ростом частоты $F_{p}$ волны (при $F_{p}>2 \mathrm{kHz}$ ) уменьшаются;

4) при $\varepsilon_{m} \geq \varepsilon_{m, t h} \approx 4 \cdot 10^{-6}$ имеет место насыщение нелинейных потерь и проявляется тенденция к насыщению сдвига резонансных частот, при которой $\Delta F_{n l, p}\left(\varepsilon_{m}\right) / F_{p} \propto \varepsilon_{m}^{1 / 2}$, при этом $\mu_{n l, p}\left(\varepsilon_{m}\right)$ практически не зависит от частоты $F_{p}$, а $\Delta F_{n l, p}\left(\varepsilon_{m}\right) / F_{p}$ слабо уменьшается с ее ростом.

Аналитическое описание нелинейных потерь и сдвига резонансных частот проведено в рамках реологической модели и уравнения состояния микронеоднородной среды с учетом насыщения гистерезисной нелинейности и релаксации ее дефектов. Из сравнения теоретических и экспериментальных амплитудно-частотных зависимостей эффектов АЗВТ удалось подобрать функцию распределения $N=N\left(\xi, W, a, b, \gamma_{0}\right)$ дефектов по параметрам $\xi, W, a, b$ и $\gamma_{0}$, отвечающим за нелинейные потери, сдвиг резонансных частот и их насыщение, и определить значения эффективных параметров $a_{\mathrm{eff}}\left(F_{p}\right)$ и $b_{\text {eff }}\left(F_{p}\right)$ гистерезисной нелинейности отожженной меди и их зависимость от частоты. Для объяснения зависимости $\Delta F_{n l, p}\left(\varepsilon_{m}\right) / F_{p} \propto \varepsilon_{m}^{1 / 2}$ полагалось, что при $\varepsilon_{m}>\varepsilon_{m, t h} \approx 4 \cdot 10^{-6}$ в уравнении состояния $\sigma=\sigma(\varepsilon)$ имеет место нелинейная поправка следующего вида: $\Delta \sigma(\varepsilon) \propto v_{1,2}\left(|\varepsilon|-\varepsilon_{m, t h}\right)^{3 / 2} h\left(|\varepsilon|-\varepsilon_{m, t h}\right)$.

В заключение отметим, что закономерности нелинейных волновых процессов в разных кристаллических твердых телах, как правило, различны, в связи с чем установление амплитудно-частотных зависимостей эффектов АЗВТ можно использовать для определения физических механизмов гистерезисной нелинейности кристаллов и развития их нелинейной акустической диагностики.

\section{Финансирование работы}

Работа поддержана РФФИ (грант N20-02-00215A).

\section{Конфликт интересов}

Авторы заявляют, что у них нет конфликта интересов.

\section{Список литературы}

[1] K.A. Naugol'nykh, L.A. Ostrovsky. Nonlinear Wave Processes in Acoustics (Cambridge University Press, 1998)

[2] V.E. Nazarov, A.V. Radostin. Nonlinear Wave Processes in Elastic Micro-inhomogeneous Solids (Wiley, 2015)

[3] L.K. Zarembo, V.A. Krasilnikov. Sov. Phys. Usp., 13, 778 (1970).

[4] L.D. Landau, E.M. Lifshiz. Course of Theoretical Physics. Theory of Elasticity (Pergamon Press, NY., 1986)

[5] M.A. Isakovich. General Acoustics (Cambridge, 1973)

[6] В.Е. Назаров, С.Б. Кияшко. Изв. вузов. Радиофизика, 62 (5), 390 (2019). [V.E. Nazarov, S.B. Kiyashko. Radiophys. Quantum Electron., 62 (5), 348 (2019).]

[7] Н.Н. Давиденков. ЖТФ, 8 (6), 483 (1938).

[8] T.A. Read. Phys. Rev., 58, 371 (1940).

[9] A. Granato, K. Lücke. J. Appl. Phys., 27, 583 (1956).

[10] Ультразвуковые методы исследования дислокащий. Сб. статей. Пер. с англ. и нем. под ред. Л.Г. Меркулова. (ИИЛ, M., 1963)

[11] Application to quantum and solid state physics, in Physical Acoustics and Methods, Ed. by Warren P. Mason, (Academic Press, NY., London, 1966), v. 4, Part A.

[12] Д. Ниблетт, Дж. Уилкс. УФН, 80 (1), 125 (1963).

[13] S. Asano. J. Phys. Soc. Jap., 29 (4), 952 (1970).

[14] А.Б. Лебедев. ФТТ, 41 (7), 1214 (1999).

[15] В.П. Левин, В.Б. Проскурин. Дислокационная неупругость в металлах (Наука, М., 1993)

[16] A.S. Novick. Phys. Rev., 80 (2), 249 (1950).

[17] S. Takahachi. J. Appl. Phys., 11 (12), 1253 (1956).

[18] D.N. Beshers. J. Appl. Phys., 30 (2), 252 (1959).

[19] L.A. Kamentsky. Thesis (Cornel University, AFOSR-TN-56425, 1956).

[20] U. Hiki. J. Phys. Soc. Jap., 13 (8), 1138 (1958).

[21] I.J. Teutonico, A.V. Granato, K. Lucke. J. Appl. Phys., 35 (1), 220 (1964).

[22] K. Lucke, A.V. Granato, I.J. Teutonico. J. Appl. Phys., 39 (11), 5181 (1968).

[23] A.V. Granato, K. Lucke. J. Appl. Phys., 52 (12), 7136 (1981).

[24] P. Peguin, H.K. Birnbaum. J. Appl. Phys., 39 (9), 4428 (1968).

[25] D.G. Blair, T.S. Hutchinson, D.H. Rogers. Canadian J. Phys., 49 (6), 633 (1971).

[26] G. Gremaud. Mater. Sci. Eng. A, 521-522, 12 (2009).

[27] С.Б. Кустов, С.Н. Голяндин, А.В. Никифоров, Б.К. Кардашев. ФТТ, 31 (2), 260 (1989).

[28] С.Н. Голяндин, С.Б. Кустов. ФТТ, 34 (12), 3763 (1992).

[29] С.Н. Голяндин, С.Б. Кустов. ФТТ, 34 (12), 3771 (1992).

[30] С.Н. Голяндин, С.Б. Кустов. ФТТ, 37 (11), 3248 (1995).

[31] S.N. Golyandin, S.B. Kustov. J. Alloys Compaunds, 211-212, 164 (1994).

[32] S. Kustov, G. Gremaud, W. Benoit, Y. Nisino, S. Asano. J. Appl. Phys., 85 (3), 1444 (1999).

[33] С.Н. Голяндин, С.Б. Кустов, К.В. Сапожников, Ю.А. Емельянов, А.Б. Синапи, С.П. Никаноров, У.Х. Робинсон. ФТТ, 40 (10), 1839 (1998).

[34] К.В. Сапожников, С.Н. Голяндин, С.Б. Кустов. ФТТ, 52 (1), 43 (2010). 
[35] В.Е. Назаров, С.Б. Кияшко. ЖТФ, $84(3), 1$ (2014). [V.E. Nazarov, S.B. Kiyashko. Tech. Phys., 59 (3), 311 (2014). DOI: $10.1134 / \mathrm{S} 1063784214030207]$

[36] Р. Хоникомб. Пластическая деформация металлов (Мир, М., 1972) [R.W.K. Honeycombe. The plastic deformation of metals, пер. с англ. под ред. Б.Я. Любова (Cambridge, 1968)]

[37] Д.В. Сивухин. Атомная и ядерная физика (Физматлит, M., 2002), т. 5.

[38] J.C. Swartz, J. Weertman. J. Appl. Phys., 32 (10), 1860 (1961).

[39] D. Gelli. J. Appl. Phys., 33 (4), 1547 (1962). 